\title{
Neural Responses to Naturalistic Clips of Behaving Animals in Two Different Task Contexts
}

\author{
Samuel A. Nastase ${ }^{1,2 *}$, Yaroslav O. Halchenko ${ }^{1}$, Andrew C. Connolly ${ }^{3}$, M. Ida Gobbini ${ }^{1,4}$ \\ and James V. Haxby ${ }^{1}$ \\ ${ }^{1}$ Department of Psychological and Brain Sciences, Center for Cognitive Neuroscience, Dartmouth College, Hanover, NH, \\ United States, ${ }^{2}$ Princeton Neuroscience Institute, Princeton University, Princeton, NJ, United States, ${ }^{3}$ Department of \\ Neurology, Geisel School of Medicine at Dartmouth, Hanover, NH, United States, ${ }^{4}$ Dipartimento di Medicina Specialistica, \\ Diagnostica e Sperimentale, Medical School, University of Bologna, Bologna, Italy
}

Keywords: action understanding, attention, categorization, fMRI, multivariate pattern analysis (MVPA), natural vision, open data

OPEN ACCESS

Edited by:

Lars Muckli,

University of Glasgow

United Kingdom

Reviewed by:

Natasha Sigala,

University of Sussex, United Kingdom

Marius Peelen,

Radboud University, Netherlands

*Correspondence:

Samuel A. Nastase

sam.nastase@gmail.com

Specialty section:

This article was submitted to

Perception Science,

a section of the journal

Frontiers in Neuroscience

Received: 20 February 2018

Accepted: 24 April 2018

Published: 15 May 2018

Citation:

Nastase SA, Halchenko YO,

Connolly AC, Gobbini MI and

Haxby JV (2018) Neural Responses to

Naturalistic Clips of Behaving Animals in Two Different Task Contexts.

Front. Neurosci. 12:316. doi: 10.3389/fnins.2018.00316
The human brain rapidly deploys semantic information during perception to facilitate our interaction with the world. These semantic representations are encoded in the activity of distributed populations of neurons (Haxby et al., 2001; McClelland and Rogers, 2003; Kriegeskorte et al., 2008b) and command widespread cortical real estate (Binder et al., 2009; Huth et al., 2012). The neural representation of a stimulus can be described as a location (i.e., response vector) in a high-dimensional neural representational space (Kriegeskorte and Kievit, 2013; Haxby et al., 2014). This resonates with behavioral and theoretical work describing mental representations of objects and actions as being organized in a multidimensional psychological space (Attneave, 1950; Shepard, 1958, 1987; Edelman, 1998; Gärdenfors and Warglien, 2012). Current applications of this framework to neural representation (e.g., Kriegeskorte et al., 2008b) often implicitly assume that these neural representational spaces are relatively fixed and context-invariant. In contrast, earlier work emphasized the importance of attention and task demands in actively reshaping representational space (Shepard, 1964; Tversky, 1977; Nosofsky, 1986; Kruschke, 1992). A growing body of work in both electrophysiology (e.g., Sigala and Logothetis, 2002; Sigala, 2004; Cohen and Maunsell, 2009; Reynolds and Heeger, 2009) and human neuroimaging (e.g., Hon et al., 2009; Jehee et al., 2011; Brouwer and Heeger, 2013; Çukur et al., 2013; Sprague and Serences, 2013; Harel et al., 2014; Erez and Duncan, 2015; Nastase et al., 2017) has suggested mechanisms by which behavioral goals dynamically alter neural representation.

Here we present functional MRI data measured while participants freely viewed brief naturalistic video clips of animals behaving in their natural environments (Nastase et al., 2017). Participants performed a 1-back category repetition detection task requiring them to attend to either animal behavior or taxonomy. There are several benefits to using dynamic, naturalistic stimuli. They convey rich perceptual and semantic information (Bartels and Zeki, 2004; Huth et al., 2012) and more fully sample neural representational space than conventional stimuli (Haxby et al., 2014). Furthermore, natural vision paradigms have greater ecological validity (Felsen and Dan, 2005), and dynamic stimuli have been shown to drive reliable neural responses across individuals (Hasson et al., 2010; Haxby et al., 2011). Data of this kind are amenable to multivariate pattern analyses (e.g., classification or representational similarity analyses; Norman et al., 2006; Kriegeskorte et al., 2008a) or forward encoding analyses using visual (e.g., Nishimoto et al., 2011), semantic (e.g., Huth et al., 2012), or neuromorphic models 
(e.g., recurrent or deep convolutional neural networks; Güçlü and van Gerven, 2015). These data are intended to provide a test bed for investigating object and action representation, as well as how task demands alter the neural representation of complex stimuli and their semantic qualities.

Twelve right-handed adults (seven female; mean age $=25.4$ years, $S D=2.6$, range $=21-31$ ) with normal or correctedto-normal vision were sampled from the Dartmouth College community to participate in the experiment. Participants reported no history of psychiatric or neurological disorders. All participants provided written, informed consent prior to participating in the study in compliance with the Committee for the Protection of Human Subjects at Dartmouth College, including a provision for data to be shared with other researchers around the world or on a publicly available data archive. The study was approved by the Institutional Review Board of Dartmouth College, and participants received monetary compensation for their participation. All data were collected between June 1 and September 6, 2013.

We implemented a full factorial repeated measures design (Fisher, 1935) comprising five taxonomic categories, four behavioral categories, and two tasks. The five taxonomic categories were primates, ungulates, birds, reptiles, and insects. The four behavioral categories were eating, fighting, running, and swimming. Crossing the taxonomy and behavior factors yielded 20 total taxonomy-behavior conditions. The animal taxonomy (i.e., object/form category) and behavior (i.e., action/motion category) factors were chosen as these are thought to rely on somewhat distinct, relatively well-studied neural pathways (Giese and Poggio, 2003; Connolly et al., 2012; Oosterhof et al., 2013; Sha et al., 2015; Wurm and Lingnau, 2015). The taxonomic and behavioral categories roughly correspond to intermediate levels of noun and verb hierarchies (Rosch, 1975; Fellbaum, 1990). We designed the experiment under the assumption that the stimulus dimensions conveying taxonomic and behavioral information are not integral (i.e., producing facilitation or interference across factors; Garner and Felfoldy, 1970). However, this may not hold in practice; for example, some taxonomic features may be necessary for behavior categorization, and certain taxa (e.g., birds) may interfere with the recognition of certain behaviors (e.g., running). While the taxonomy and behavior factors are fully crossed at the category level, it is not feasible to orthogonalize lower-level correlates (e.g., motion energy, the specific animal performing each action) in natural vision paradigms.

Each of the 20 taxonomy-behavior conditions comprised two unique $2 \mathrm{~s}$ video clips, as well as horizontally flipped versions of each clip for 80 visually unique stimuli in total. Video clip stimuli were sampled from nature documentaries (Life, Life of Mammals, Microcosmos, Planet Earth) and high-resolution YouTube videos. Video clips were edited using the free FFmpeg software package for handling multimedia files (https://www.ffmpeg.org). Stimuli were back-projected onto a screen located at the back of the scanner bore using a Panasonic PT-D4000U projector and viewed via a mirror mounted on the head coil. Video clips subtended a visual angle of $\sim 16.5^{\circ}$ horizontally and $\sim 11^{\circ}$ vertically. Stimuli were presented using PsychoPy (v1.76.00; http://www.psychopy.org; Peirce, 2007; RRID:SCR_006571).
In designing the experiment, we adopted a condition-rich ungrouped-events design (Kriegeskorte et al., 2008a). Each trial consisted of a $2 \mathrm{~s}$ video clip presented without sound followed by a $2 \mathrm{~s}$ fixation period for a trial onset asynchrony of $4 \mathrm{~s}$ (Figure 1A). Each of the 80 stimuli was presented once each run. This type of design has been argued to be particularly efficient for characterizing the pairwise distances between neural response patterns (Aguirre, 2007; Kriegeskorte et al., 2008a). When convolved with a hemodynamic response function, this design matrix will yield highly overlapping response predictors (Figure 1B). The response magnitude for each condition can be recovered using a conventional regression model (e.g., Nastase et al., 2017), or regularized regression can be used to predict responses based on an explicit model of stimulus features (e.g., Nishimoto et al., 2011). Each of the 80 unique stimuli can be treated as a separate condition (Kriegeskorte et al., 2008a), or 20 conditions can be defined at the category level by collapsing across the four exemplar clips per taxonomy-behavior condition (Nastase et al., 2017).

In addition to the 80 stimuli, each run included four taxonomy repetition events, four behavior repetition events, and four null fixation events. This resulted in 92 events per run, plus an additional $12 \mathrm{~s}$ fixation appended to the beginning and end of each run, for a total run duration of $392 \mathrm{~s}(\sim 6.5 \mathrm{~min})$. Ten unique runs were created and run order was counterbalanced across participants using a Latin square (Fisher, 1935). Each run was constructed in the following way. First, a pseudorandom trial order containing all 80 stimuli and no taxonomic or behavioral category repetitions was assembled. Second, eight additional stimuli were inserted at particular locations in the trial order to induce four taxonomic category repetition events and four behavioral category repetitions events. Note that in one run an error occurred where a behavior repetition event was inserted that interrupted a previously inserted taxonomic repetition event; this error went unnoticed during data collection but is explicitly noted in text files accompanying the data. These sparse repetition events were inserted such that a repetition event of both types occurred within each quarter of the run. We ensured that the same clip exemplar (or the horizontally mirrored version) never occurred twice consecutively, and that for each taxonomic or behavioral category repetition, the repetition stimulus varied along the other dimension. Finally, four $2 \mathrm{~s}$ null events comprising only a fixation cross were inserted at pseudorandom locations in the trial order to effect temporal jittering. One of the four null fixation events occurred each quarter of the run and did not interrupt repetition events. This resulted in an overall scan duration of $\sim 65 \mathrm{~min}$.

Prior to scanning, participants were verbally familiarized with the task and the categories. At the beginning of each run, participants received written instructions indicating that they should pay attention to either taxonomy or behavior and press the button only when they observed a category repetition of that type. Participants were informed that they should ignore repetitions of the unattended type during that run. Button presses were only required for the sparse repetition events (not for nonrepetitions) and the same button was used for repetitions of both types. Although responses were collected for repetition events to 
A

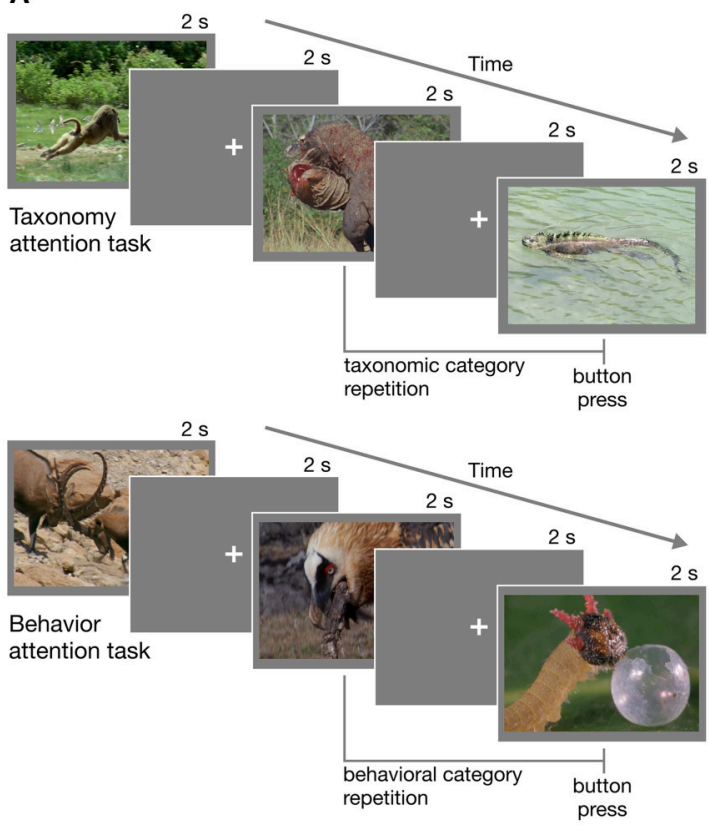

B

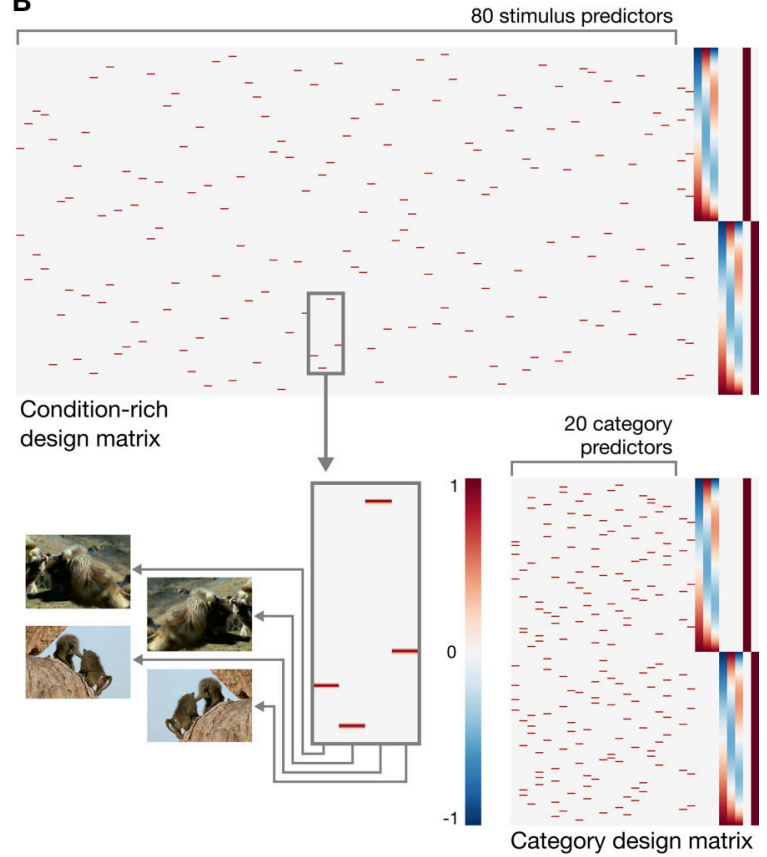

FIGURE 1 | Experimental design. (A) Schematic of the rapid event-related design for both taxonomy attention and behavior attention task conditions. In the taxonomy attention task, participants were instructed to press a button if they observed a taxonomic category repetition (e.g., two consecutive clips depicting reptiles; upper). In the behavior attention task, participants were instructed to press a button if they observed a behavioral category repetition (e.g., two consecutive clips depicting animals eating; lower). (B) Two example design matrices for predicting hemodynamic responses to the clips over the course of two runs with the taxonomy attention task. In the condition-rich design, each of 80 visually unique stimuli receives a separate predictor (following Kriegeskorte et al., $2008 \mathrm{a}$; upper), while in the category design, the four exemplar clips per taxonomy-behavior condition are collapsed to form 20 category predictors (following Nastase et al., 2017 ; lower). Hypothesized neural responses are convolved with a simple hemodynamic response function (Cohen, 1997). In this simple example, nuisance regressors for taxonomy and behavior repetition events, first- through third-order Legendre polynomials, and run constants are appended to each design matrix. Figures were created using Matplotlib (https://matplotlib.org; Hunter, 2007; RRID:SCR_008624) and seaborn (https://seaborn.pydata.org; Waskom et al., 2016).

ensure task compliance, this task was not intended to robustly measure response latencies. We use the term attention loosely here, as performing the 1-back category repetition detection task also requires categorization, working memory, and motor processes. Participants were instructed to maintain fixation only during the fixation periods, and freely viewed the video clip stimuli (cf. Çukur et al., 2013). Behavioral responses for repetition events were collected using a single two-button Lumina LS-PAIR response pad (Cedrus, San Pedro, CA) held in the right hand.

All functional and structural images were acquired using a $3 \mathrm{~T}$ Philips Intera Achieva MRI scanner (Philips Healthcare, Bothell, WA; RRID:SCR_008656) with a 32-channel phased-array head coil. Functional, blood-oxygenation-level-dependent (BOLD) images were acquired in an interleaved fashion using gradientecho echo-planar imaging with a SENSE parallel imaging factor of 2 (Pruessmann et al., 1999): TR/TE $=2000 / 35 \mathrm{~ms}$, flip angle $=90^{\circ}$, resolution $=3 \mathrm{~mm}^{3}$ isotropic, matrix size $=80$ $\times 80, \mathrm{FoV}=240 \times 240 \mathrm{~mm}, 42$ transverse slices with full brain coverage and no gap. At the beginning of each run, two dummy scans were acquired to allow for signal stabilization. Ten runs were collected for each participant, each consisting of 196 functional volumes totaling $392 \mathrm{~s}(\sim 6.5 \mathrm{~min})$ in duration. At the end of each session, a T1-weighted structural scan was acquired using a high-resolution single-shot MPRAGE sequence:
$\mathrm{TR} / \mathrm{TE}=8.2 / 3.7 \mathrm{~ms}$, flip angle $=8^{\circ}$, resolution $=0.9375 \times$ $0.9375 \times 1.0 \mathrm{~mm}^{3}$ voxels, matrix size $=256 \times 256, \mathrm{FoV}=240$ $\times 240 \times 220 \mathrm{~mm}^{3}$. The BOLD signal reflects metabolic demands and serves as a rough proxy for neural activity (primarily local field potentials; Logothetis et al., 2001).

All data have been curated and organized according to the Brain Imaging Data Structure (BIDS) standards (Gorgolewski et al., 2016), and are freely available via the OpenNeuro repository (https://openneuro.org; Poldrack and Gorgolewski, 2017). Data are version-controlled and conveniently accessible using the DataLad data distribution (http://datalad.org; Halchenko et al., 2017; RRID:SCR_003932, RRID:SCR_003931) from their original location at http://datasets.datalad.org/? dir=/labs/haxby/attention, as well as from OpenNeuro at https://openneuro.org/datasets/ds000233 (RRID:SCR_005031). According to the BIDS conventions, data are stored in separate directories for each participant alongside the scripts used to compile and analyze the data, a descriptive text file, and a tab-separated text file describing participant demographics. Within each participant's directory, anatomical and functional images are stored in separate directories. Both anatomical and functional images are stored in compressed Neuroinformatics Informatics Technology Initiative (NIfTI-1) format (Cox et al., 2003; RRID:SCR_003141). Structural images were de-faced for 
A Behavior classification across taxa while attending to behavior
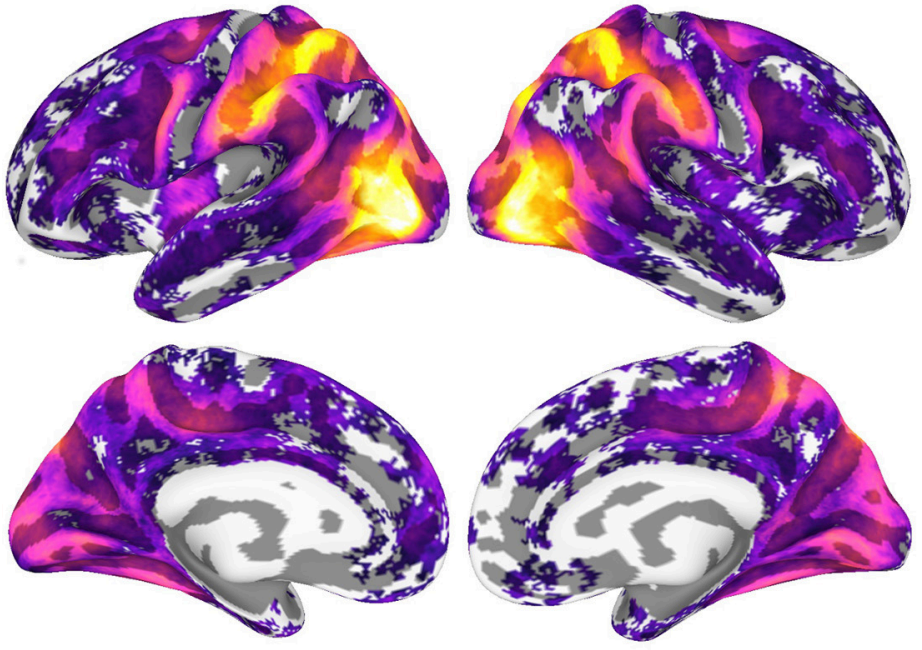

B Taxonomy classification across behaviors while attending to taxonomy
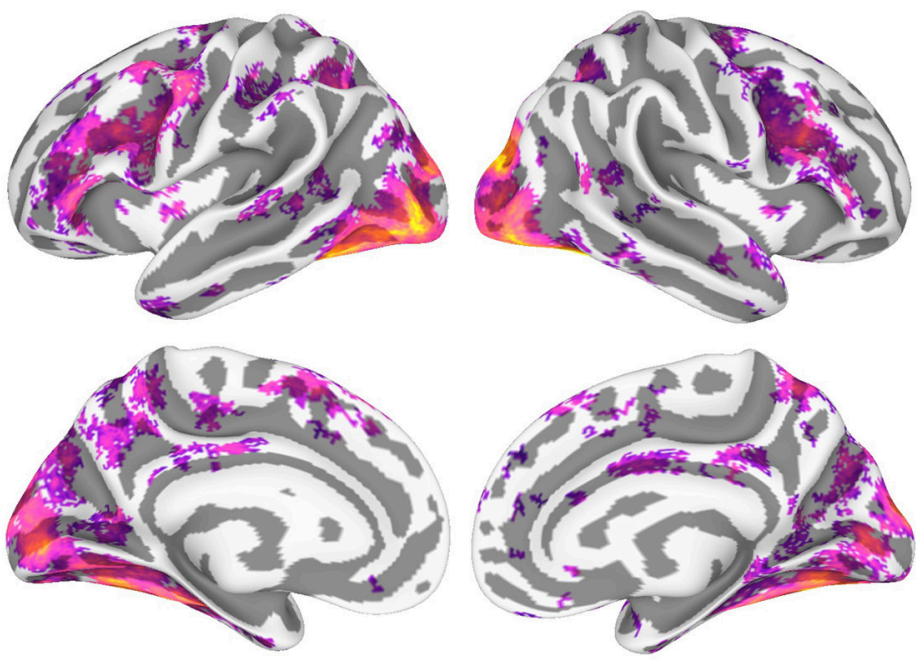
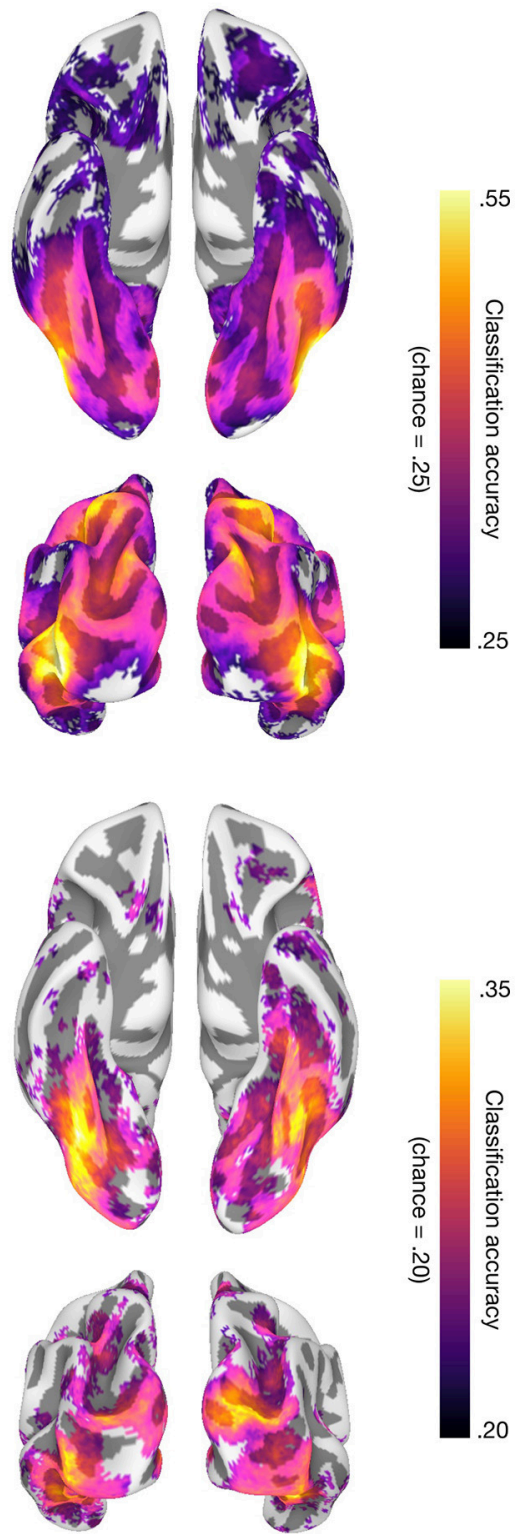

FIGURE 2 | Behavioral and taxonomic category cross-classification using surface-based searchlights. To statistically evaluate the searchlight results, we first computed a one-sample $t$-test against theoretical chance accuracy per searchlight (one-tailed test). We corrected for multiple tests by controlling the false discovery rate (FDR) at $q=0.05$ (Benjamini and Hochberg, 1995; Genovese et al., 2002). The mean classification accuracy across participants is plotted and searchlight maps are thresholded at FDR $q=0.05$. (A) Searchlight classification of behavioral categories cross-validated across taxonomic categories while participants attended to animal behavior. Theoretical chance accuracy for four-way behavioral category classification is 0.25 . The maximum mean searchlight accuracy for behavioral category classification was 0.56 in left lateral occipitotemporal cortex (inferior occipital gyrus). (B) Searchlight classification of taxonomic categories cross-validated across behavioral categories while participants attended to animal taxonomy. Theoretical chance accuracy for five-way taxonomic category classification is 0.20 . The maximum mean searchlight accuracy for taxonomic category classification was 0.36 in right ventral temporal cortex (lateral fusiform gyrus). Although we used a $t$-test here for simplicity, note that the $t$-test may yield significant $t$-values even for near-chance accuracies, and a permutation- or prevalence-based approach may be preferable in some cases (cf. Stelzer et al., 2013; Allefeld et al., 2016; Etzel, 2017). Surface vertices on the medial wall were excluded from the analysis and clusters of fewer than ten contiguous significant vertices after thresholding were excluded for visualization purposes. Surface data were visualized using SUMA (Saad et al., 2004; RRID:SCR_005927) and figures were created using GIMP (https://www.gimp.org; RRID:SCR_003182) and Inkscape (https://inkscape.org; RRID:SCR_014479).

anonymization purposes using an automated masking procedure (Hanke et al., 2014). Each functional run is accompanied by a file describing the acquisition parameters as well as a tab-separated text file describing the following for each event: the filename of the clip stimulus, the onset time, duration (2s), taxonomy-behavior condition, taxonomic category, and behavioral category of the stimulus, as well as whether the stimulus was horizontally mirrored, and whether the event was a repetition or not (and of what type). Participant-specific button presses and their associated response times are also included 
in the table. The 40 video clip stimuli (MPEG-4 files) are distributed alongside the data in keeping with fair use provisions for non-commercial scholarly research. Derived data, resulting from preprocessing or other analyses are stored separately in the top-level directory and recapitulate a similar directory structure.

Behaviorally, participants reported category repetitions with high accuracy (99\% for both tasks, as reported in Nastase et al., 2017). Although this suggests that participants allocated attention sufficiently to perform the task, it precludes investigators from relating the magnitude of attentional demands to neural responses. We did not design the experiment with a "baseline" or "no task" condition, as it is unclear what this would entail in the context of natural vision paradigms, and any claims about task demands must rely on relative differences between the two tasks.

Organizing data in the standardized BIDS format facilitates the use of portable analysis tools called BIDS Apps (Gorgolewski et al., 2017). To assess the general quality of the data, we used the MRIQC tool (v0.9.6; https:/github.com/poldracklab/ mriqc; Esteban et al., 2017a). Across all participants and runs, median temporal signal-to-noise ratio (tSNR) was 64.73 (range: 31.06-89.14), which approximates the expected tSNR given $3 \mathrm{~mm}$ isotropic voxels and 3T magnetic field strength (Triantafyllou et al., 2005), and is comparable to existing data sets (e.g., Sengupta et al., 2016). Mean framewise displacement (Power et al., 2012) was on average $0.15 \mathrm{~mm}$ (range: $0.10-0.44 \mathrm{~mm}$ ) across participants and runs, indicating fairly low head motion. The full MRIQC report is available alongside the data at OpenNeuro.org.

To verify that events were annotated correctly, we performed a simple multivariate analysis. Data were first preprocessed using the fMRIPrep BIDS App (v1.0.0-rc5; https:/github. com/poldracklab/fmriprep; Esteban et al., 2017b, in review), a Nipype-based tool (Gorgolewski et al., 2011; RRID:SCR_002502, RRID:SCR_002823). Cortical surfaces were reconstructed from anatomical scans using FreeSurfer (v6.0.0; https://surfer.nmr. mgh.harvard.edu; Dale et al., 1999; RRID:SCR_001847) and spatially normalized to the fsaverage6 template based on sulcal curvature (Fischl et al., 1999). Preprocessed data output by fMRIPrep are available at OpenNeuro.org, including volumetric and surface-based formats both in native space and normalized to standardized templates. FMRIPrep also returns as a variety of nuisance variables intended to capture head motion and physiological artifacts. Functional images were corrected for slice-timing (Cox, 1996), head motion (Jenkinson et al., 2002), and aligned to the anatomical image (Greve and Fischl, 2009). Functional data were not explicitly spatially smoothed. We then used a general linear model implemented in AFNI (v17.1.02; https:/afni.nimh.nih.gov; Cox, 1996; RRID:SCR_005927) to estimate response patterns for the 20 taxonomy-behavior conditions in each run per task. Nuisance regressors comprised framewise displacement (Power et al., 2012), the first six principal components from an automatic anatomical segmentation of cerebrospinal fluid (aCompCor; Zhang et al., 2001; Behzadi et al., 2007), and de-meaned head motion parameters and their derivatives, regressors for repetition events and button presses, as well as first- through third-order Legendre polynomials.

We then used linear support vector machines (SVMs; Boser et al., 1992; Chang and Lin, 2011; RRID:SCR_010243) in surfacebased searchlights $(10 \mathrm{~mm}$ radius; Kriegeskorte et al., 2006; Oosterhof et al., 2011) to classify taxonomic and behavioral categories. We used a leave-one-category-out cross-classification approach in both cases: to classify the five taxonomic categories, we trained SVMs on three of the four behavior categories and tested on the left-out behavior category (Figure 2A); to classify the four behavioral categories, we trained SVMs on four of the five taxonomic categories and tested on the leftout taxonomic category (Figure 2B). This approach requires that information about, e.g., behavioral categories, encoded in local response patterns generalizes across both stimuli and taxonomic categories (Kaplan et al., 2015; Nastase et al., 2016; Westfall et al., 2016). All multivariate analyses were performed using PyMVPA (v2.6.3.dev1; http://www.pymvpa.org; Hanke et al., 2009; RRID:SCR_006099) in the NeuroDebian computational environment (Debian “jessie” 8.5 GNU/Linux with NeuroDebian repositories; http://neuro.debian.net; Hanke and Halchenko, 2011; RRID:SCR_006638, RRID:SCR_004401), making heavy use of Python-based tools SciPy (https://www.scipy.org; Jones et al., 2001; RRID:SCR_008394, RRID:SCR_008058), NumPy (http://www.numpy.org; Walt et al., 2011; RRID:SCR_008633), and the IPython interactive shell (https://ipython.org; Perez and Granger, 2007; RRID:SCR_001658). All scripts used to perform these analyses are provided alongside the data. The resulting searchlight maps corroborate prior work on action and taxonomic category representation (e.g., Connolly et al., 2012; Wurm and Lingnau, 2015; Nastase et al., 2017), and demonstrate the potential utility of the data set.

\section{AUTHOR CONTRIBUTIONS}

SN, JH, AC, and MG: designed the experiment; SN: collected and analyzed the data; $\mathrm{SN}, \mathrm{YH}$, and JH: wrote the manuscript; $\mathrm{SN}$ and $\mathrm{YH}$ : curated the data for public sharing.

\section{FUNDING}

This work was supported by the National Institute of Mental Health at the National Institutes of Health (grant numbers F32MH085433-01A1 to AC; and 5R01MH075706 to JH), and the National Science Foundation (grant numbers NSF1129764 and NSF1607845 to JH).

\section{DATA AVAILABILITY STATEMENT}

The datasets generated and analyzed for this study can be found in the DataLad (http://datasets.datalad.org/?dir=/labs/haxby/ attention) and OpenNeuro (https://openneuro.org/datasets/ ds000233) repositories. Data preprocessed using fMRIPrep are available at the OpenNeuro repository. Data at each stage of analysis used in this report, including the final results, are available at the DataLad repository. 


\section{ACKNOWLEDGMENTS}

We thank Jason Gors, Kelsey G. Wheeler, Matteo Visconti di Oleggio Castello, J. Swaroop Guntupalli, Courtney Rogers, and Terry Sackett for assistance in collecting stimuli and data. We also thank Krzysztof J. Gorgolewski for providing helpful comments in an open review (http://academickarma.org/review/ jfp011q60d20).

\section{REFERENCES}

Aguirre, G. K. (2007). Continuous carry-over designs for fMRI. Neuroimage 35, 1480-1494. doi: 10.1016/j.neuroimage.2007.02.005

Allefeld, C., Görgen, K., and Haynes, J. D. (2016). Valid population inference for information-based imaging: from the second-level $t$-test to prevalence inference. Neuroimage 141, 378-392. doi: 10.1016/j.neuroimage.2016. 07.040

Attneave, F. (1950). Dimensions of similarity. Am. J. Psychol. 63, 516-556. doi: $10.2307 / 1418869$

Bartels, A., and Zeki, S. (2004). Functional brain mapping during free viewing of natural scenes. Hum. Brain Mapp. 21, 75-85. doi: 10.1002/hbm.10153

Behzadi, Y., Restom, K., Liau, J., and Liu, T. T. (2007). A component based noise correction method (CompCor) for BOLD and perfusion based fMRI. Neuroimage 37, 90-101. doi: 10.1016/j.neuroimage.2007.04.042

Benjamini, Y., and Hochberg, Y. (1995). Controlling the false discovery rate: a practical and powerful approach to multiple testing. J. R. Stat. Soc. Series B Stat. Methodol. 57, 289-300.

Binder, J. R., Desai, R. H., Graves, W. W., and Conant, L. L. (2009). Where is the semantic system? A critical review and meta-analysis of 120 functional neuroimaging studies. Cereb. Cortex 19, 2767-2796. doi: 10.1093/cercor/bhp055

Boser, B. E., Guyon, I. M., and Vapnik, V. N. (1992). “A training algorithm for optimal margin classifiers," in Proceedings of the Fifth Annual Workshop on Computational Learning Theory, ed D. Haussler (Pittsburgh, PA), 144-152.

Brouwer, G. J., and Heeger, D. J. (2013). Categorical clustering of the neural representation of color. J. Neurosci. 33, 15454-15465. doi: 10.1523/jneurosci.2472-13.2013

Chang, C.-C., and Lin, C.-J. (2011). LIBSVM: a library for support vector machines. ACM Trans. Intell. Syst. Technol. 2:27. doi: 10.1145/1961189.1961199

Cohen, M. R., and Maunsell, J. H. (2009). Attention improves performance primarily by reducing interneuronal correlations. Nat. Neurosci. 12, 1594-1600. doi: $10.1038 / \mathrm{nn} .2439$

Cohen, M. S. (1997). Parametric analysis of fMRI data using linear systems methods. Neuroimage 6, 93-103. doi: 10.1006/nimg.1997.0278

Connolly, A. C., Guntupalli, J. S., Gors, J., Hanke, M., Halchenko, Y. O., Wu, Y.C., et al. (2012). The representation of biological classes in the human brain. J. Neurosci. 32, 2608-2618. doi: 10.1523/jneurosci.5547-11.2012

Cox, R. W. (1996). AFNI: software for analysis and visualization of functional magnetic resonance neuroimages. Comput. Biomed. Res. 29, 162-173. doi: 10.1006/cbmr.1996.0014

Cox, R. W., Ashburner, J., Breman, H., Fissell, K., Haselgrove, C., Holmes, C. J., Lancaster, J. L., et al. (2003). "A (sort of) new image data format standard: NIfTI-1," in 10th Annual Meeting of the Organization for Human Brain Mapping (Budapest).

Çukur, T., Nishimoto, S., Huth, A. G., and Gallant, J. L. (2013). Attention during natural vision warps semantic representation across the human brain. Nat. Neurosci. 16, 763-770. doi: 10.1038/nn.3381

Dale, A. M., Fischl, B., and Sereno, M. I. (1999). Cortical surface-based analysis: I. Segmentation and surface reconstruction. Neuroimage 9, 179-194. doi: 10.1006/nimg.1998.0395

Edelman, S. (1998). Representation is representation of similarities. Behav. Brain Sci. 21, 449-467. doi: 10.1017/S0140525X980 01253

Erez, Y., and Duncan, J. (2015). Discrimination of visual categories based on behavioral relevance in widespread regions of frontoparietal cortex. J. Neurosci. 35, 12383-12393. doi: 10.1523/jneurosci.1134-15.2015

Esteban, O., Birman, D., Schaer, M., Koyejo, O. O., Poldrack, R. A., and Gorgolewski, K. J. (2017a). MRIQC: advancing the automatic prediction

of image quality in MRI from unseen sites. PLoS ONE 12:e0184661. doi: 10.1371/journal.pone.0184661

Esteban, O., Blair, R., Markiewicz, C. J., Berleant, S. L., Moodie, C., Feilong, M., et al. (2017b). poldracklab/fmriprep: 1.0.0-rc5. Zenedo. doi: $10.5281 /$ zenodo.996169

Etzel, J. A. (2017). "MVPA significance testing when just above chance, and related properties of permutation tests", in 2017 International Workshop on Pattern Recognition in Neuroimaging (PRNI) (Toronto, ON).

Fellbaum, C. (1990). English verbs as a semantic net. Int. J. Lexicogr. 3, 278-301. doi: $10.1093 / \mathrm{ijl} / 3.4 .278$

Felsen, G., and Dan, Y. (2005). A natural approach to studying vision. Nat. Neurosci. 8, 1643-1646. doi: 10.1038/nn1608

Fischl, B., Sereno, M. I., Tootell, R. B., and Dale, A. M. (1999). High-resolution intersubject averaging and a coordinate system for the cortical surface. Hum. Brain Mapp. 8, 272-284. doi: 10.1002/(SICI)1097-0193(1999)8:4<272::AID-HBM10>3.0.CO;2-4

Fisher, R. A. (1935). The Design of Experiments. Edinburgh: Oliver and Boyd.

Gärdenfors, P., and Warglien, M. (2012). Using conceptual spaces to model actions and events. J. Semant. 29, 487-519. doi: 10.1093/jos/ffs007

Garner, W. R., and Felfoldy, G. L. (1970). Integrality of stimulus dimensions in various types of information processing. Cogn. Psychol. 1, 225-241. doi: 10.1016/0010-0285(70)90016-2

Genovese, C. R., Lazar, N. A., and Nichols, T. (2002). Thresholding of statistical maps in functional neuroimaging using the false discovery rate. Neuroimage 15, 870-878. doi: 10.1006/nimg.2001.1037

Giese, M. A., and Poggio, T. (2003). Neural mechanisms for the recognition of biological movements. Nat. Rev. Neurosci. 4, 179-192. doi: 10.1038/ nrn1057

Gorgolewski, K. J., Alfaro-Almagro, F., Auer, T., Bellec, P., Capotăa, M., Chakravarty, M. M., Churchill, N. W., et al. (2017). BIDS apps: improving ease of use, accessibility, and reproducibility of neuroimaging data analysis methods. PLoS Comput. Biol. 13:e1005209. doi: 10.1371/journal.pcbi.10 05209

Gorgolewski, K. J., Auer, T., Calhoun, V. D., Craddock, R. C., Das, S., Duff, E. P., Flandin, G., et al. (2016). The brain imaging data structure, a format for organizing and describing outputs of neuroimaging experiments. Sci. Data 3:160044. doi: 10.1038/sdata.2016.44

Gorgolewski, K., Burns, C. D., Madison, C., Clark, D., Halchenko, Y. O. Waskom, M. L., et al. (2011). Nipype: a flexible, lightweight and extensible neuroimaging data processing framework in python. Front. Neuroinform. 5:13. doi: $10.3389 /$ fninf.2011.00013

Greve, D. N., and Fischl, B. (2009). Accurate and robust brain image alignment using boundary-based registration. Neuroimage 48, 63-72. doi: 10.1016/j.neuroimage.2009. 06.060

Güçlü, U., and van Gerven, M. A. (2015). Deep neural networks reveal a gradient in the complexity of neural representations across the ventral stream. J. Neurosci. 35, 10005-10014. doi: 10.1523/jneurosci.5023-14.2015

Halchenko, Y. O., Hanke, M., Poldrack, B., Solanky, D. S., Alteva, G., Gors, J., et al. (2017). datalad/datalad 0.9.1. doi: 10.5281/zenodo.1000098

Hanke, M., and Halchenko, Y. O. (2011). Neuroscience runs on GNU/Linux. Front. Neuroinform. 5:8. doi: 10.3389/fninf.2011.00008

Hanke, M., Baumgartner, F. J., Ibe, P., Kaule, F. R., Pollmann, S., Speck, O., et al. (2014). A high-resolution 7-Tesla fMRI dataset from complex natural stimulation with an audio movie. Sci. Data 1:140003. doi: 10.1038/sdata. 2014.3

Hanke, M., Halchenko, Y. O., Sederberg, P. B., Hanson, S. J., Haxby, J. V., and Pollmann, S. (2009). PyMVPA: a Python toolbox for multivariate pattern analysis of fMRI data. Neuroinformatics 7, 37-53. doi: 10.1007/s12021-008-9041-y 
Harel, A., Kravitz, D. J., and Baker, C. I. (2014). Task context impacts visual object processing differentially across the cortex. Proc. Natl. Acad. Sci. U.S.A. 111, E962-E971. doi: 10.1073/pnas.1312567111

Hasson, U., Malach, R., and Heeger, D. J. (2010). Reliability of cortical activity during natural stimulation. Trends Cogn. Sci. 14, 40-48. doi: 10.1016/j.tics.2009.10.011

Haxby, J. V., Connolly, A. C., and Guntupalli, J. S. (2014). Decoding neural representational spaces using multivariate pattern analysis. Annu. Rev. Neurosci. 37, 435-456. doi: 10.1146/annurev-neuro-062012170325

Haxby, J. V., Gobbini, M. I., Furey, M. L., Ishai, A., Schouten, J. L., and Pietrini, P. (2001). Distributed and overlapping representations of faces and objects in ventral temporal cortex. Science 293, 2425-2430. doi: 10.1126/science.10 63736

Haxby, J. V., Guntupalli, J. S., Connolly, A. C., Halchenko, Y. O., Conroy, B. R., Gobbini, M. I., et al. (2011). A common, high-dimensional model of the representational space in human ventral temporal cortex. Neuron 72, 404-416. doi: 10.1016/j.neuron.2011.08.026

Hon, N., Thompson, R., Sigala, N., and Duncan, J. (2009). Evidence for long-range feedback in target detection: detection of semantic targets modulates activity in early visual areas. Neuropsychologia 47, 1721-1727. doi: 10.1016/j.neuropsychologia.2009.02.011

Hunter, J. D. (2007). Matplotlib: a 2D graphics environment. Comput. Sci. Eng. 9, 90-95. doi: $10.1109 /$ mcse.2007.55

Huth, A. G., Nishimoto, S., Vu, A. T., and Gallant, J. L. (2012). A continuous semantic space describes the representation of thousands of object and action categories across the human brain. Neuron 76, 1210-1224. doi: 10.1016/j.neuron.2012.10.014

Jehee, J. F., Brady, D. K., and Tong, F. (2011). Attention improves encoding of task-relevant features in the human visual cortex. J. Neurosci. 31, 8210-8219. doi: 10.1523/jneurosci.6153-09.2011

Jenkinson, M., Bannister, P., Brady, M., and Smith, S. (2002). Improved optimization for the robust and accurate linear registration and motion correction of brain images. Neuroimage 17, 825-841. doi: 10.1006/nimg.2002.1132

Jones, E., Oliphant, T., and Peterson, P. (2001). SciPy: Open Source Scientific Tools for Python. Available online at: http://www.scipy.org

Kaplan, J. T., Man, K., and Greening, S. G. (2015). Multivariate cross-classification: applying machine learning techniques to characterize abstraction in neural representations. Front. Hum. Neurosci. 9:151. doi: 10.3389/fnhum.2015. 00151

Kriegeskorte, N., and Kievit, R. A. (2013). Representational geometry: integrating cognition, computation, and the brain. Trends Cogn. Sci. 17, 401-412. doi: 10.1016/j.tics.2013.06.007

Kriegeskorte, N., Goebel, R., and Bandettini, P. (2006). Information-based functional brain mapping. Proc. Natl. Acad. Sci. U.S.A. 103, 3863-3868. doi: $10.1073 /$ pnas. 0600244103

Kriegeskorte, N., Mur, M., and Bandettini, P. A. (2008a). Representational similarity analysis-connecting the branches of systems neuroscience. Front. Syst. Neurosci. 2:4. doi: 10.3389/neuro.06.004.2008

Kriegeskorte, N., Mur, M., Ruff, D. A., Kiani, R., Bodurka, J., Esteky, H., et al. (2008b). Matching categorical object representations in inferior temporal cortex of man and monkey. Neuron 60, 1126-1141. doi: 10.1016/j.neuron.2008.10.043

Kruschke, J. K. (1992). ALCOVE: an exemplar-based connectionist model of category learning. Psychol. Rev. 99, 22-44. doi: 10.1037/0033-295X. 99.1.22

Logothetis, N. K., Pauls, J., Augath, M., Trinath, T., and Oeltermann, A. (2001). Neurophysiological investigation of the basis of the fMRI signal. Nature 412, 150-157. doi: $10.1038 / 35084005$

McClelland, J. L., and Rogers, T. T. (2003). The parallel distributed processing approach to semantic cognition. Nat. Rev. Neurosci. 4, 310-322. doi: $10.1038 /$ nrn 1076

Nastase, S. A., Connolly, A. C., Oosterhof, N. N., Halchenko, Y. O., Guntupalli, J. S., Visconti di Oleggio Castello, M., et al. (2017). Attention selectively reshapes the geometry of distributed semantic representation. Cereb. Cortex 27, 4277-4291. doi: 10.1093/cercor/bhx138
Nastase, S. A., Halchenko, Y. O., Davis, B., and Hasson, U. (2016). "Crossmodal searchlight classification: methodological challenges and recommended solutions," in 2016 International Workshop on Pattern Recognition in Neuroimaging (PRNI) (Trento).

Nishimoto, S., Vu, A. T., Naselaris, T., Benjamini, Y., Yu, B., and Gallant, J. L. (2011). Reconstructing visual experiences from brain activity evoked by natural movies. Curr. Biol. 21, 1641-1646. doi: 10.1016/j.cub.2011.08.031

Norman, K. A., Polyn, S. M., Detre, G. J., and Haxby, J. V. (2006). Beyond mind-reading: multi-voxel pattern analysis of fMRI data. Trends Cogn. Sci. 10, 424-430. doi: 10.1016/j.tics.2006.07.005

Nosofsky, R. M. (1986). Attention, similarity, and the identification-categorization relationship. J. Exp. Psychol. Gen. 115, 39-57. doi: 10.1037/0096-3445.1 15.1.39

Oosterhof, N. N., Tipper, S. P., and Downing, P. E. (2013). Crossmodal and actionspecific: neuroimaging the human mirror neuron system. Trends Cogn. Sci. 17, 311-318. doi: 10.1016/j.tics.2013.04.012

Oosterhof, N. N., Wiestler, T., Downing, P. E., and Diedrichsen, J. (2011). A comparison of volume-based and surface-based multi-voxel pattern analysis. Neuroimage 56, 593-600. doi: 10.1016/j.neuroimage.2010. 04.270

Peirce, J. W. (2007). PsychoPy-Psychophysics software in Python. J. Neurosci. Methods 162, 8-13. doi: 10.1016/j.jneumeth.2006. 11.017

Perez, F., and Granger, B. E. (2007). IPython: a system for interactive scientific computing. Comput. Sci. Eng. 9, 21-29. doi: 10.1109/mcse.2007.53

Poldrack, R. A., and Gorgolewski, K. J. (2017). OpenfMRI: open sharing of task fMRI data. Neuroimage 8, 259-261. doi: 10.1016/j.neuroimage.2015. 05.073

Power, J. D., Barnes, K. A., Snyder, A. Z., Schlaggar, B. L., and Petersen, S. E. (2012). Spurious but systematic correlations in functional connectivity MRI networks arise from subject motion. Neuroimage 59, 2142-2154. doi: 10.1016/j.neuroimage.2011.10.018

Pruessmann, K. P., Weiger, M., Scheidegger, M. B., Boesiger, P., and Others (1999). SENSE: sensitivity encoding for fast MRI. Magn. Reson. Med. 42, 952-962. doi: 10.1002/(SICI)1522-2594(199911)42:5<952::AID-MRM16>3.0.CO;2-S

Reynolds, J. H., and Heeger, D. J. (2009). The normalization model of attention. Neuron 61, 168-185. doi: 10.1016/j.neuron.2009.01.002

Rosch, E. (1975). Cognitive representations of semantic categories. J. Exp. Psychol. Gen. 104, 192-233. doi: 10.1037/0096-3445.104.3.192

Saad, Z. S., Reynolds, R. C., Argall, B., Japee, S., and Cox, R. W. (2004). "SUMA: an interface for surface-based intra- and inter-subject analysis with AFNI," in 2004 2nd IEEE International Symposium on Biomedical Imaging: Macro to Nano (Arlington, VA).

Sengupta, A., Kaule, F. R., Guntupalli, J. S., Hoffmann, M. B., Häusler, C., Stadler, J., et al. (2016). A studyforrest extension, retinotopic mapping and localization of higher visual areas. Sci. Data 3:160093. doi: 10.1038/sdata. 2016.93

Sha, L., Haxby, J. V., Abdi, H., Guntupalli, J. S., Oosterhof, N. N., Halchenko, Y. O., et al. (2015). The animacy continuum in the human ventral vision pathway. $J$. Cogn. Neurosci. 27, 665-678. doi: 10.1162/jocn_a_00733

Shepard, R. N. (1958). Stimulus and response generalization: tests of a model relating generalization to distance in psychological space. J. Exp. Psychol. 55, 509-523. doi: $10.1037 / \mathrm{h} 0042354$

Shepard, R. N. (1964). Attention and the metric structure of the stimulus space. J. Math. Psychol. 1, 54-87. doi: 10.1016/0022-2496(64) 90017-3

Shepard, R. N. (1987). Toward a universal law of generalization for psychological science. Science 237, 1317-1323. doi: 10.1126/science.36 29243

Sigala, N. (2004). Visual categorization and the inferior temporal cortex. Behav. Brain Res. 149, 1-7. doi: 10.1016/S0166-4328(03)00224-9

Sigala, N., and Logothetis, N. K. (2002). Visual categorization shapes feature selectivity in the primate temporal cortex. Nature 415, 318-320. doi: $10.1038 / 415318 \mathrm{a}$

Sprague, T. C., and Serences, J. T. (2013). Attention modulates spatial priority maps in the human occipital, parietal and frontal cortices. Nat. Neurosci. 16, 1879-1887. doi: 10.1038/nn.3574 
Stelzer, J., Chen, Y., and Turner, R. (2013). Statistical inference and multiple testing correction in classification-based multi-voxel pattern analysis (MVPA): random permutations and cluster size control. Neuroimage 65, 69-82. doi: 10.1016/j.neuroimage.2012.09.063

Triantafyllou, C., Hoge, R. D., Krueger, G., Wiggins, C. J., Potthast, A., Wiggins, G. C., et al. (2005). Comparison of physiological noise at $1.5 \mathrm{~T}, 3 \mathrm{~T}$ and $7 \mathrm{~T}$ and optimization of fMRI acquisition parameters. Neuroimage 26, 243-250. doi: 10.1016/j.neuroimage.2005.01.007

Tversky, A. (1977). Features of similarity. Psychol. Rev. 84, 327-352. doi: 10.1037/0033-295X.84.4.327

Walt, S., van der, Colbert, S. C., and Varoquaux, G. (2011). The NumPy array: a structure for efficient numerical computation. Comput. Sci. Eng. 13, 22-30. doi: $10.1109 /$ mcse.2011.37

Waskom, M. L., Botvinnik, O., O'Kane, D., Hobson, P., Halchenko, Y. O., Lukauskas, S., Cole, J. B., et al. (2016). seaborn: v0.7.1. doi: $10.5281 /$ zenodo. 54844

Westfall, J., Nichols, T. E., and Yarkoni, T. (2016). Fixing the stimulusas-fixed-effect fallacy in task fMRI. Wellcome Open Res. 1:23. doi: 10.12688/wellcomeopenres.10298.2
Wurm, M. F., and Lingnau, A. (2015). Decoding actions at different levels of abstraction. J. Neurosci. 35, 7727-7735. doi: 10.1523/jneurosci.0188-15.2015

Zhang, Y., Brady, M., and Smith, S. (2001). Segmentation of brain MR images through a hidden Markov random field model and the expectation-maximization algorithm. IEEE Trans. Med. Imaging 20, 45-57. doi: $10.1109 / 42.906424$

Conflict of Interest Statement: The authors declare that the research was conducted in the absence of any commercial or financial relationships that could be construed as a potential conflict of interest.

Copyright (c) 2018 Nastase, Halchenko, Connolly, Gobbini and Haxby. This is an open-access article distributed under the terms of the Creative Commons Attribution License (CC BY). The use, distribution or reproduction in other forums is permitted, provided the original author(s) and the copyright owner are credited and that the original publication in this journal is cited, in accordance with accepted academic practice. No use, distribution or reproduction is permitted which does not comply with these terms. 